\title{
Likelihood of repeat abortion in a Swedish cohort according to the choice of post-abortion contraception: a longitudinal study
}

Helena Kilander, Siw Alehagen, Linnea Svedlund, Karin Westlund, Johan Thor and Jan

Brynhildsen

Linköping University Post Print

\section{Tweet}

N.B.: When citing this work, cite the original article.

Original Publication:

Helena Kilander, Siw Alehagen, Linnea Svedlund, Karin Westlund, Johan Thor and Jan Brynhildsen, Likelihood of repeat abortion in a Swedish cohort according to the choice of postabortion contraception: a longitudinal study, 2016, Acta Obstetricia et Gynecologica Scandinavica, (95), 5, 565-571.

http://dx.doi.org/10.1111/aogs.12874

Copyright: Wiley: 12 months

http://eu.wiley.com/WileyCDA/

Postprint available at: Linköping University Electronic Press

http://urn.kb.se/resolve?urn=urn:nbn:se:liu:diva-128136 
Received Date : 15-Nov-2015

Accepted Date : 25-Jan-2016

Article type : Original Research Article

\section{Likelihood of repeat abortion in a Swedish cohort according to the choice of post-abortion contraception: a longitudinal study}

Running title: Post abortion contraception and odds of repeat abortion

Helena Kilander ${ }^{1,2}$, Siw Alehagen ${ }^{1}$, Linnea Svedlund ${ }^{1}$, Karin Westlund ${ }^{3}$, Johan Thor $^{4} \&$ Jan Brynhildsen ${ }^{5}$

${ }^{1}$ Division of Nursing Science, Department of Medicine and Care, Faculty of Health Sciences, Linköping University, Linköping.

${ }^{2}$ Department of Obstetrics and Gynecology in Eksjö, County Council of Jönköping.

${ }^{3}$ Department of Obstetrics and Gynecology, Norrköping.

${ }^{4}$ Jönköping Academy for Improvement of Health and Welfare, Jönköping University, Jönköping.

${ }^{5}$ Department of Obstetrics and Gynecology and Department of Clinical and Experimental Medicine, Linköping University, Linköping, Sweden. 


\section{Correspondence to:}

Helena Kilander

Kvinnokliniken Höglandssjukhuset, 57581 Eksjö, Sweden

E-mail: helena.kilander@rjl.se

\section{Conflicts of Interest statement}

Jan Brynhildsen has been reimbursed by Merck Sharpe \& Dohme (MSD), Sweden, for running educational programs and giving lectures. JB has also been paid by Bayer AB, Sweden, and Actavis, for giving lectures. Other co-authors have no conflicts of interest to report. 
Introduction: Despite high access to contraceptive services, $42 \%$ of the women who seek an abortion in Sweden have a history of previous abortion(s). The reasons for this high repeat abortion rate remain obscure. The objective of this study was to study the choice of contraceptive method after abortion and related odds of repeat abortions within three to four years. Material and methods: This is a retrospective cohort study based on a medical record review at three hospitals in Sweden. We included 987 women who had an abortion during 2009. We reviewed medical records from the date of the index abortion until the end of 2012 to establish the choice of contraception following the index abortion and the occurrence of repeat abortions. We calculated odds ratios (OR) with $95 \%$ confidence intervals $(\mathrm{CI})$. Results: While $46 \%$ of the women chose oral contraceptives, $34 \%$ chose long-acting reversible contraceptives (LARC). LARC was chosen more commonly by women with a previous pregnancy, childbirth and/or abortion. During the follow-up period, $24 \%$ of the study population requested one or more repeat abortion(s). Choosing LARC at the time of the index abortion was associated with fewer repeat abortions compared with choosing oral contraceptives ( $13 \%$ versus $26 \%$, OR 0.36 ; $95 \%$ CI 0.24-0.52). Sub-dermal implant was as effective as intrauterine device in preventing repeat abortions beyond three years. Conclusions: Choosing LARC was associated with fewer repeat abortions over more than three years of follow-up.

\section{Keywords}

Long-acting reversible contraception, post-abortion contraception, repeat abortion, Subdermal implant, termination of pregnancy.

\section{Abbreviations}

OR odds ratio,

CI confidence intervals,

LARC long-acting reversible contraception,

OC oral contraceptives,

LNG-IUS levonorgestrel intrauterine systems,

IUD intrauterine device. 

Choosing long-acting reversible contraception (LARC) post-abortion was associated with considerably fewer repeat abortions compared to choosing other contraceptive methods among women in Sweden.

38

39

40

41

42

43

44

45

46

47

48

49

50

51

52

53

54

55

56

57 


\section{Introduction}

Many unwanted pregnancies occur in spite of contraceptive use, probably due to inconsistent or incorrect use of the contraceptives (1-4). A French study reported that one-third of women undergoing an abortion chose to use the same contraceptive method after the abortion as before. Half of these women reported receiving a prescription for an oral contraceptive (OC) (4). Both prescribers and users may overestimate the effectiveness of OC (1, 3-5). Women undergoing an abortion seem, however, particularly willing to switch to more effective, nonuser dependent, contraceptive methods such as long acting reversible contraception (LARC) (3-6).

Intrauterine contraception and sub-dermal implants (LARC), unlike OC, does not require the user to remember to take it each day or in conjunction with intercourse (7). Previous studies have found that use of LARC after an abortion leads to a greater reduction in the risk of further unintended pregnancies and repeat abortions compared with use of other contraceptive methods. Few studies have included choice and use of sub-dermal implants post abortion and studies with longer follow-up time are lacking (8-12). A high initial cost for levonorgestrel intrauterine systems (LNG-IUS) and sub-dermal implants is, however, a challenge affecting the choice of contraceptive method (13-14).

There are approximately 37000 induced abortions in Sweden every year, corresponding to a rate of almost 21 per 1000 women aged 15-49 years (15). In Sweden, $42 \%$ of all women who undergo an abortion have had at least one previous abortion (15). Women are free to undergo an abortion until the 18th week of pregnancy. Thereafter, an abortion requires approval by the National Board of Health and Welfare. Medical abortions constitute $88 \%$ of all abortions.

Women are offered a follow-up appointment three to four weeks after a medical abortion (15). Usually, initiation of LARC is arranged during this appointment, whereas women who undergo surgical abortion often have LARC inserted immediately. Contraceptive counselling is free for women of all ages and a number of hormonal contraceptives (including sub-dermal implants and LNG-IUS) are subsidized up to the age of 25 (16). In connection with an abortion, either a gynaecologist or a midwife carries out contraceptive counselling (17). 
In Sweden, approximately $25 \%$ of all fertile women use an intrauterine device (IUD), while 5$8 \%$ use implants (18). It is unclear why, despite these comparatively large proportions, $42 \%$ of women who have had an abortion undergo repeat abortions (15). We therefore studied the choice of contraceptive method after abortion and the related odds of repeat abortions within three to four years.

\section{Material and methods}

This was a retrospective cohort study based on the review of medical records. The study was performed in departments of Obstetrics and Gynecology at one university hospital, one central hospital and one district hospital in southeast Sweden. We included all women with a Social Security Number (i.e. permanent residents of Sweden) who sought an abortion during 2009 at these hospitals and had a diagnosis of 'unwanted pregnancy' (z.64.0 in ICD-10). Women were excluded if the review showed that the diagnosis was incorrect at the index visit (e.g. the record indicated an spontaneous abortion), if the woman chose to continue the pregnancy, or if medical interventions preventing pregnancy, such as sterilization or hysterectomy, occurred during the follow-up period. Women were also excluded if the induced abortion occurred because of maternal disease or due to fetal malformations, since these women might wish to become pregnant soon again. The study was approved by the Regional Ethical Review Board in Linköping (\#2013/145-31; date of approval: 24 April 2013.)

The medical records were reviewed from the date of the index abortion during 2009 to the 31st of December 2012. Data were manually extracted from the medical records using a standardized form. Local administrators de-identified all records before some of the authors reviewed them. The data abstraction procedure and form was pilot tested on 30 records, leading to a few adjustments. These 30 pilot medical records were then included in the study. Three authors collected data (HK, LS and KW) and a fourth author (JB) acted as an adjudicator in case of questions and disagreements.

From the index abortion records, we extracted data on women's age, number of previous pregnancies (including all previous deliveries, abortions and miscarriages), current Chlamydia infection and use of an interpreter. The contraceptive method chosen was noted either if the women started the method immediately or the record noted that they planned to start later on. 
Moreover, we collected data on any medical history that could constitute a contraindication for a specific contraceptive choice, on the method used for the index abortion and on any complications associated with the index abortion. We included information concerning the choice of contraceptive method up until four weeks after the index abortion.

If no contraception was chosen, it was recorded as "No chosen method". LARC was defined as sub-dermal implants and IUDs, which included Copper-IUDs and LNG-IUS.

Each woman's medical record was reviewed for repeat abortions at the same hospital from the time of the index abortion until the end of 2012. We were not able to trace women outside their local hospital, throughout Sweden, based on their Social Security Number since no individual based abortion register exists, and there are multiple different medical record systems across the country. The authors relied on the collaborating hospitals' administrators to identify relevant records, and only received de-identified copies of records from them, to protect patient privacy.

A repeat abortion was defined as termination of one or more new pregnancies during the follow-up period. A total of 1574 women had the diagnosis z64.0. From this group, 1,395 records were identified of women who actually had an abortion during 2009 at the study hospitals and met the inclusion criteria for review. Of these records, 408 were excluded because the patient had moved out of the area of each hospital $(n=337)$ or had more than one contraceptive listed $(n=26)$ or had no information regarding contraceptive choice in the record at the time of the index abortion $(n=45)$. The remaining 987 records were included in this study.

For power calculation, we hypothesized that the choice of LARC after an index abortion would be associated with fewer repeat abortions within three to four years compared with other contraceptive options. Based on a previous study we expected $50 \%$ fewer repeat abortions among LARC users compared to users of other methods. We assumed that $30 \%$ of the women would choose LARC (8). To reach $80 \%$ power to identify this difference at a 0.05 significance level, we needed to include 705 women (medical records). Since there was considerable uncertainty in this estimate, and assuming that a number of women would have no follow-up, we decided to double this number. 
Statistical analyses were performed using IBM SPSS statistics version 20 (IBM Corp., Armonk, NY, USA). In the statistical analysis, repeat abortion during follow-up was considered as both a continuous and a categorical variable. Using the Student's t-test we compared the characteristics of the women who chose LARC with those of the women who chose other contraceptive options. We used the Chi-squared test for calculating p-values and presented the results as odds ratios (OR) with $95 \%$ confidence intervals (CI) for the categorical variables contraceptive method, repeat abortion and abortion method. Using a logistic regression model we adjusted for the potential confounding factors age, previous pregnancy, childbirth and abortions. We used Kaplan Meier's survival test to estimate the proportions of women not having a repeat abortion according to their chosen contraceptive methods from the time of the index abortion until the first repeat abortion.

\section{Results}

Thirty eight percent of the women $(n=375)$ had undergone one or more previous abortion(s) before the index abortion. Twenty-seven women (2.7\%) were diagnosed with a Chlamydia infection. Few women $(n=57)$ had a medical or family history noted in the records that could constitute a contraindication for use of a specific contraceptive method. Twenty-four women used an interpreter at the time of the index abortion, and in 17 additional cases, language difficulties were noted in the records when no interpreter had been present.

In all, 239 women $(24 \%)$ returned for one or more repeat abortions at the same hospital as their index abortion during follow-up; 53 of them (5\%) underwent two or more abortions. The median time until the first repeat abortion among the 239 women was 17 months.

Women who chose LARC had a significantly higher number of previous pregnancies (2.6 versus 1.7 on average), of children (1.5 versus 1.0$)$ and of previous abortions ( 0.8 versus 0.6 ). Those who underwent a surgical abortion chose LARC more frequently than women who underwent a medical abortion (42\% versus $32 \%, \mathrm{p}<0.03)$ (Table 1$)$.

Women were less likely to choose LARC than OC (P=0.02, OR: 0.74 CI: 0.55-0.98). OC was prescribed to $46 \%$ of the women; $28 \%$ chose combined oral contraceptives and $18 \%$ chose progestin only pills. LARC was chosen by $34 \%$ of the women at the index abortion. Women 
who chose progestin only pills, barrier methods or chose no method at all had similar rates of repeat abortions (33-34\%, Table 2).

A smaller proportion of women who chose LARC underwent repeat abortions than did women who chose other contraceptive methods (Tables 2, 3), or had no chosen method. Choice of sub-dermal implant yielded the same level of effectiveness as choice of IUD in preventing repeat abortions beyond three years (Table 2). In a logistic regression model including choice of LARC, previous pregnancies, childbirths, abortions and age, choice of LARC remained strongly associated with a decreased risk of repeated abortion (Table 3). Furthermore, analysing year by year after the index abortion, the choice of LARC was associated with fewer repeat abortions compared with all other choices (OR $\leq 0.36 ; 95 \% \mathrm{CI}$ 0.19-0.64).

Choice of LARC was associated with fewer repeat abortions also when compared only with choice of OC (table 3). Over the follow-up period, $90 \%$ of the women who chose LARC at the time of the index abortion avoided having any repeat abortion, while only $75 \%$ of the women who chose OC did so - a proportion similar to that of women who chose no contraceptive method at all (Figure 1).

Among women who chose to use an IUD at the index abortion $(n=257)$, we found no difference in the odds of repeat abortion between those who underwent a surgical abortion and those who underwent a medical abortion.

\section{Discussion}

We found that $24 \%$ of the women returned for abortion(s) within three to four years following an index abortion. The odds of repeat abortion were significantly lower for women who chose LARC compared to all other options, thus confirming our hypothesis. The choice of subdermal implant was as effective as IUD in preventing repeat abortions over three to four years, corroborating the few previous studies following women beyond two years $(11,21)$. Previous studies $(11,20)$, while pointing in the same direction, included shorter follow-up periods than the present study. This study expands the body of evidence for the effectiveness and comparative advantage of LARC including implants, which have rarely been included to the same extent in previous studies, in preventing repeat abortion over 3-4 years compared to 
other contraceptive methods.

220 Even though the proportion of women who chose LARC was comparatively high, at 34\%, 221 there is room for increasing use of LARC to further prevent repeat abortions. A Finnish 222 register study had a long follow-up time after abortion (49 months) but only few women used 223 an implant (8). We followed 987 women for more than three years. Moreover, the study 224 reflects an "everyday situation" and confirms that the benefits of LARC found in (efficacy) intervention studies $(8-9,11)$ are replicable in ordinary clinical settings. As the vast majority of abortion care in Sweden is provided by public health care services, we likely included and followed the vast majority of the women who underwent abortion in the catchment areas of the hospitals. Furthermore, we included all women, whether they underwent surgical or medical abortion, which previous studies $(8-9,20)$ have not done. Only 12 women were excluded due to abortion for medical reasons, and five women were excluded because they had a hysterectomy during the follow-up. Consequently, this could not be considered a source of bias that affects the results.

We have only evaluated the post-abortion choices of contraceptives (as documented in the medical record) rather than actual use of contraception, since such data are much more difficult to acquire; there, the medical record does not suffice as a data source. It is likely some women did not use the method they had chosen (according to the medical record) and others discontinued its use. We actually do not know how many subsequently discontinued the method during our follow-up period. Not starting use or discontinuing use are probably the major causes of pregnancy and repeat abortion among the women who chose LARC (according to their record) rather than becoming pregnant when actually using LARC.

As in previous studies $(3,19)$ OC showed to be less effective, but the most frequently selected contraceptive method, following an abortion in our study population. LARC offered better protection against repeat abortion than other options, yet was chosen by a minority of women at the time of their index abortion. There are many possible explanations for why women did not choose the more effective LARC over OC. After a medical abortion, use of LARC requires an additional visit to initiate the method, whereas it can be introduced concomitantly with surgical abortion (20). Therefore, as might be expected, a greater proportion of women in our study who had a surgical abortion chose to use LARC. Simplifying initiation and use of LARC post-abortion is an important issue. Sääw and co-workers showed that an IUD could be safely inserted five to ten days after a medical abortion (22) and recently it has been described 
that sub-dermal implants can be inserted at the time of mifepristone intake during a medical abortion (23).

Lack of knowledge and inadequate clinical training of health care professionals may hinder women from using LARC (14, 24-26). While midwives and gynaecologists who provide contraceptive counselling in Sweden are expected to have these skills, it is unclear to what extent they actually do support LARC during such counselling.

Poor bleeding control is a well-known reason for discontinuation of contraceptive use. This may explain why the women in our study more frequently chose combined hormonal contraceptives than LARC (26). Some prescribers may not recommend LARC to nulliparous women due to a common misconception that these women prefer to use OC $(27,28)$. By contrast, however, adolescents might benefit the most from using LARC instead of OC (10). In our study, LARC was chosen more often by women with a previous pregnancy, childbirth and /or abortion, perhaps due to the increased motivation by the women and their counsellors to change to a more effective, non-user-dependent, method. Here, too, we see room for increased use of LARC, also among nulliparous women.

Financial factors may have influenced the women's choice of contraception in this study and in previous studies $(8,20)$. Implants and LNG-IUS are associated with a high initial cost (14, 24). In an American study where all methods were offered for free for three years, $70 \%$ of women who were given structured contraceptive counselling chose LARC (29). More broadly, previous studies have shown a relationship between repeat abortion and socioeconomic factors $(1,4,30)$. While we were not able to address this important issue here, since data on patients' socioeconomic status were not consistently available in the medical records, we note the potential relationship between the cost of different contraceptive methods and the socioeconomic gradient in the risk of unwanted pregnancy. Good access to LARC for all women, regardless of socioeconomic status, could prevent unwanted pregnancies and improve sexual and reproductive health (10).

In conclusion, this study shows that more women chose OC than LARC even though LARC was associated with considerably fewer repeat abortions over 3-4 years compared to choice of other contraceptive options. Sub-dermal implant was as effective as IUD in preventing repeat abortions. These findings highlight the importance, not least at the time of an abortion, of providing evidence based information to women who might not know the large differences in effectiveness of the different methods. More research regarding experiences and perceptions 
283 among both healthcare professionals and women could reveal possible obstacles to more

284 effective contraceptive counselling. Interventional studies could test the feasibility of

285 promoting LARC to prevent repeat abortions.

\section{Acknowledgements}

287 The authors are grateful to the administrators of each participating hospital, who produced the 288 de-identified medical records, and to the statistician Mats Fredriksson at Linköping Academic 289 Centre for statistical advice and support.

\section{Funding}

291 This study was funded by grants from Futurum - the academy for healthcare, in the Jönköping 292 County Council, and the Medical Research Council of Southeast Sweden (FORSS). 


\section{References}

2961.

Trussell J. Contraceptive failure in the United States. Contraception.2011; 83:

$297397-404$.

298 2. Eisenberg DL, Secura GM, Madden TE, Allsworth JE, Zhao Q, Peipert JF.

299 Knowledge of contraceptive effectiveness. Am J Obstet Gynecol. 2012; 206:479 e.1-9.

3003 3. Moreau C, Trussell J, Desfreres J, Bajos N. Patterns of contraceptive use before

301 and after an abortion: results from a nationally representative survey of women undergoing an

302 abortion in France. Contraception.2010; 82:337-44.

303 4. Trussell J. Understanding contraceptive failure. Best Pract Res Clin Obstet

304 Gynaecol. 2009; 23:199-209.

305 5. Trussell J, Henry N, Hassan F, Prezioso A, Law A, Filonenko A. Burden of

306 unintended pregnancy in the United States: potential savings with increased use of long-acting

307 reversible contraception. Contraception. 2013; 87:154-61.

308 6. Kero A, Lalos A. Increased contraceptive use one year post-abortion. Hum

309 Reprod. 2005; 20:3085-90.

3107 Grimes DA. Forgettable contraception. Contraception.2009; 80: 497-9.

3118 8. Heikinheimo O, Gissler M, Suhonen S. Age, parity, history of abortion and

312 contraceptive choices affect the risk of repeat abortion. Contraception.2008; 78: 149-54.

$3139 . \quad$ Rose SB, Lawton BA. Impact of long-acting reversible contraception on return

314 for repeat abortion. Am J Obstet Gynecol. 2012; 206:37 e.1-6.

315 10. Winner B, Peipert JF, Zhao Q, Buckel C, Madden T, Allsworth JE, et al.

316 Effectiveness of long-acting reversible contraception. N Engl J Med. 2012; 366: 1998-2007.

317 11. Cameron ST, Glasier A, Chen ZE, Johnstone A, Dunlop C, Heller R. Effect of

318 contraception provided at termination of pregnancy and incidence of subsequent termination

319 of pregnancy. BJOG. 2012; 119:1074-80.

320 12. Gemzell-Danielsson K, Inki P, Heikinheimo O. Recent developments in the

321 clinical use of the levonorgestrel-releasing intrauterine system. Acta Obstet Gynecol

322 Scand. 2011; 90:1177-88.

323 13. Falk G, Brynhildsen J, Ivarsson AB. Contraceptive counselling to teenagers at

324 abortion visits--a qualitative content analysis. Eur J Contracept Reprod Health Care. 2009

$325 ; 14: 357-64$. 
Contraceptive CHOICE Project: reducing barriers to long-acting reversible contraception. Am J Obstet Gynecol. 2010; 203:115 e1-7. induced abortions 2014. [In Swedish: Abortstatistik 2014] [cited 3 Nov 2015]; Available from: https://www.socialstyrelsen.se/publikationer2015/2015-9-4 16.

Danielsson M, Berglund T, Forsberg M, Larsson M, Rogala C, Tyden T. Sexual and reproductive health: Health in Sweden: The National Public Health Report 2012. Chapter 9. Scandinavian journal of public health. 2012; 40 (9 Suppl):176-96.

Svensk förening för Ostetrik och Gynekologi [Swedish Society of Obstetrics and Gynecology]. Medical guidelines for induced abortion. [In Swedish: Medicinska riktlinjer för inducerad abort, FARG. 2009 ] [cited 3 Nov 2015]; Available from: https://www.sfog.se/media/51367/medicinska_riktlinjer_f_r_inducerad_abort.pdf 18. Socialstyrelsen [The National board for Health and Welfare].Differences in costs between different types of contraceptives.Problems and proposals for action. [In Swedish: Skillnader i kostnader mellan olika typer av preventivmedel. Problem och åtgärdsförslag inom oförändrad kostnadsram. 2005] ISBN 91-85482-19-6 uptake of long-acting reversible methods of contraception among women presenting for abortion. Med J Aust. 2014; 201:412-6. IUDs, implants and DMPA reduces repeat pregnancy within 1 year in a New York City practice. Contraception. 2014; 89:103-8. levonorgestrel implants reduces the incidence of births and abortions at 2 years and beyond. Contraception. 2015; 92:17-25.

352 22. Sääv I, Stephansson O, Gemzell-Danielsson K. Early versus delayed insertion of 353 intrauterine contraception after medical abortion - a randomized controlled trial. PLoS One. $354 \quad 2012 ; 7: \mathrm{e} 48948$

355 23. Barros Pereira I, Carvalho RM, Graça LM. Intra-

356 abortion contraception with etonogestrel subdermal implant. Eur J Obstet Gynecol Reprod 357 Biol. 2015; 185:33-5. 
359 Postabortion Contraception: Qualitative Interviews on Counselling and Provision of Long-

360 Acting Reversible Contraceptive Methods. Persp on sexual and reprod health. 2012; 44:100-6.

$36125 . \quad$ Blumenthal PD, Voedisch A, Gemzell-Danielsson K. Strategies to prevent

362 unintended pregnancy: increasing use of long-acting reversible contraception. Hum Reprod

363 Update. 2011; 17:121-37.

$36426 . \quad$ Speidel JJ, Harper CC, Shields WC. The potential of long-acting reversible

365 contraception to decrease unintended pregnancy. Contraception. 2008; 78:197-200.

366 27. Russo JA, Miller E, Gold MA. Myths and misconceptions about long-acting

367 reversible contraception (LARC). J Adolesc Health. 2013; 52(4 Suppl):S14-21.

368 28. Baldwin MK, Edelman AB. The effect of long-acting reversible contraception

369 on rapid repeat pregnancy in adolescents: a review. J Adolesc Health. 2013; 52(4 Suppl):S47-

37053.

$37129 . \quad$ Mestad R, Secura G, Allsworth JE, Madden T, Zhao Q, Peipert JF. Acceptance

372 of long-acting reversible contraceptive methods by adolescent participants in the

373 Contraceptive CHOICE Project. Contraception. 2011; 84:493-8.

374 30. Fathalla MF, Sinding SW, Rosenfield A, Fathalla MM. Sexual and reproductive 375 health for all: a call for action. Lancet. 2006; 368:2095-100. 


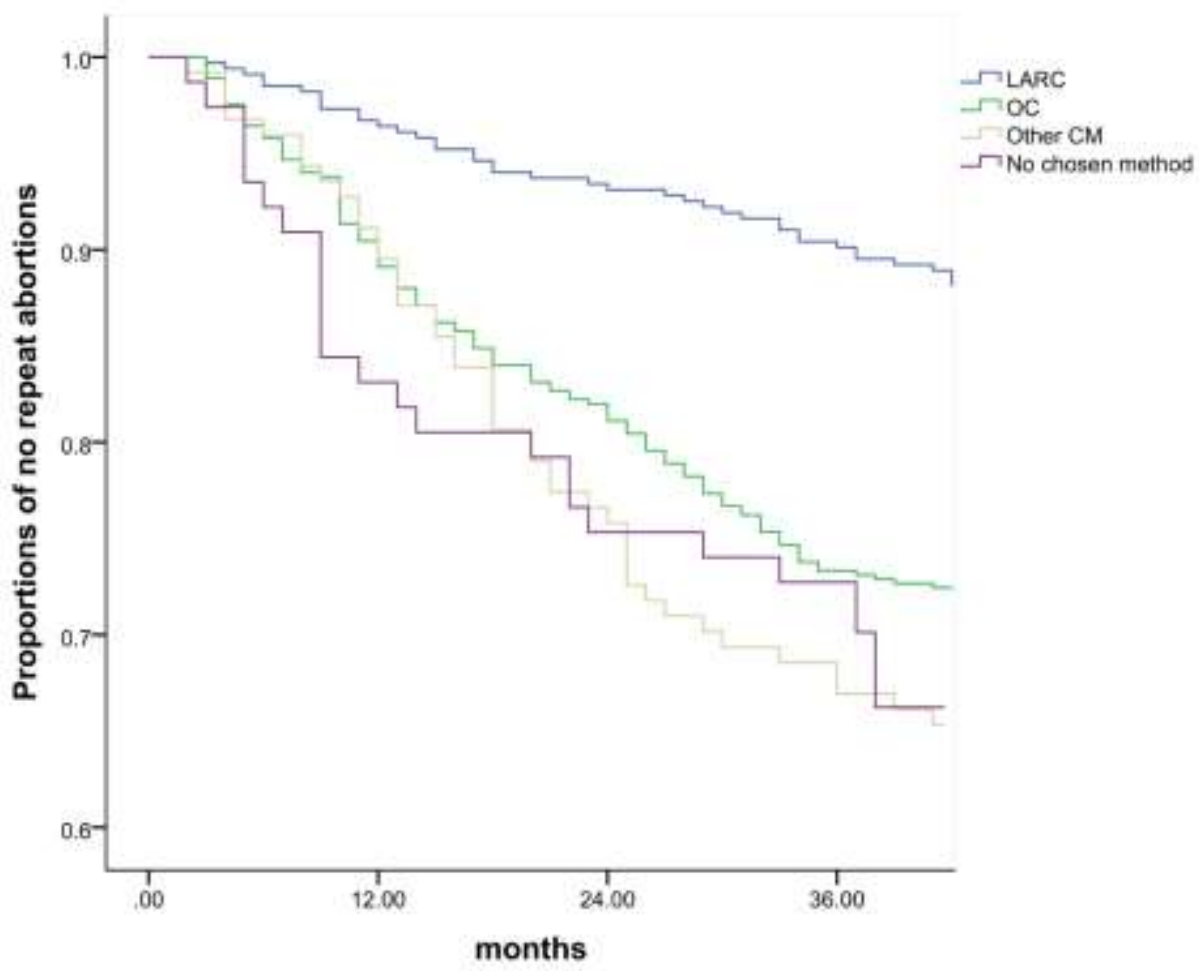

381

382 Figure 1. Probability of not having a repeat abortion according to chosen contraceptive

383 method. Calculated from the date of the index abortion until the first repeat abortion.

384 LARC, long-acting reversible contraception; OC, oral contraceptives; CM, contraceptive 385 methods.

386

387

388 


\begin{tabular}{|l|l|l|l|l|}
\hline Characteristics & $\begin{array}{l}\text { Study-population } \\
\mathrm{n}=987\end{array}$ & $\begin{array}{l}\text { No LARC a } \\
\mathrm{n}=653\end{array}$ & $\begin{array}{l}\text { LARC b } \\
\mathrm{n}=334\end{array}$ & P-value \\
\hline $\begin{array}{l}\text { Age } \\
\text { Mean (SD) }\end{array}$ & $27.7(7.9)$ & $26.9(7.7)$ & $29.3(8.1)$ & $0.09^{\mathrm{c}}$ \\
\hline $\begin{array}{l}\text { Previous } \\
\text { pregnancies } \\
\text { Mean (SD) }\end{array}$ & $2.0(2.1)$ & $1.7(2.0)$ & $2.6(2.2)$ & $0.01^{\mathrm{c}}$ \\
\hline $\begin{array}{l}\text { Previous } \\
\text { childbirth } \\
\text { Mean (SD) }\end{array}$ & $1.1(1.2)$ & $1.0(1.2)$ & $1.5(1.3)$ & $0.01^{\mathrm{c}}$ \\
\hline $\begin{array}{l}\text { Previous } \\
\text { abortions } \\
\text { Mean (SD) }\end{array}$ & $0.6(1.0)$ & $0.6(0.9)$ & $0.8(1.1)$ & $0.003^{\mathrm{c}}$ \\
\hline $\begin{array}{l}\text { Medical abortion/ } \\
\text { Surgical abortion }\end{array}$ & $803(81)$ & $546(68)$ & $257(32)$ & $0.030^{\mathrm{d}}$ \\
$\mathrm{n}(\%)$ & $184(19)$ & $77(42)$ & \\
\hline
\end{tabular}

393
Table 1. Characteristics of the study population divided by choice of Long Acting Reversible Contraception (LARC) or no LARC at the index abortion.
${ }^{a}$ No LARC: Combined oral contraceptives; Progestin only pills; Injections; The vaginal contraceptive ring; The transdermal contraceptive patch; Barrier method; Choice of no contraceptive method in the record.

${ }^{\mathrm{b}}$ LARC: Copper intrauterine device ; Levonorgestrel intrauterine system; Sub-dermal implants. ${ }^{\mathrm{c}}$ Students t-test.

${ }^{\mathrm{d}}$ Chi-square test - choice of LARC after medical versus surgical abortion. Missing values: No missing values in the variable age. Min/max 42-97 missing values in the other variables. 
Table 2. Choice of contraceptive method at the time of the index abortion, and number of repeat abortions during the follow-up period.

\begin{tabular}{|l|l|l|l|l|}
\hline Contraceptive method & Number of women & \multicolumn{2}{l|}{$\begin{array}{l}\text { Number of women undergoing one or } \\
\text { more repeat abortions during the follow- } \\
\text { up period }\end{array}$} \\
\hline & n (\%) & any (\%) & $\begin{array}{l}\text { 1 } \\
\text { n (\%) }\end{array}$ & $\begin{array}{l}\text { n (\%) } \\
\text { n }\end{array}$ \\
\hline No LARC & $653(66)$ & $197(30)$ & $155(24)$ & $42(6)$ \\
\hline Combined oral contraceptives & $277(28)$ & $72(26)$ & $54(19)$ & $18(7)$ \\
\hline Progestin only pill & $174(18)$ & $56(32)$ & $48(27)$ & $8(5)$ \\
\hline Ring/ Patch ${ }^{\text {a }}$ & $52(5)$ & $20(38)$ & $15(29)$ & $5(9)$ \\
\hline Barrier method & $42(4)$ & $14(33)$ & $10(24)$ & $4(9)$ \\
\hline Injection & $30(3)$ & $9(30)$ & $9(30)$ & 0 \\
\hline No chosen method ${ }^{b}$ & $78(8)$ & $26(33)$ & $19(24)$ & $7(9)$ \\
\hline LARC ${ }^{c}$ & $334(34)$ & $42(13)$ & $31(9)$ & $11(4)$ \\
\hline Copper intrauterine device & $159(16)$ & $19(12)$ & $13(8)$ & $6(4)$ \\
\hline Levonorgestrel intrauterine system & $98(10)$ & $12(12)$ & $11(11)$ & $1(1)$ \\
\hline Sub-dermal Implant & $77(8)$ & $11(14)$ & $7(9)$ & $4(5)$ \\
\hline Total & $987(100 \%)$ & $239(24 \%)$ & $186(19)$ & $53(5)$ \\
\hline & & & & \\
\hline
\end{tabular}

07

${ }^{\mathrm{a}}$ Ring/Patch $=$ the vaginal contraceptive ring/ the transdermal contraceptive patch. ${ }^{\mathrm{b}}$ No chosen method= Choice of no contraceptive method in the record ${ }^{\mathrm{c}}$ LARC=Long Acting Reversible Contraception 
Table 3. Choice of contraceptive method and odds of repeat abortion(s).

413

414

\begin{tabular}{|c|c|c|c|}
\hline Contraceptive methods & Women & $\begin{array}{l}\text { Odds of repeat } \\
\text { abortion }(s)^{b}\end{array}$ & $\begin{array}{l}\text { Adjustments for potential } \\
\text { confounding factors }{ }^{c}\end{array}$ \\
\hline Comparing groups & (n) & $(\mathrm{OR} / \mathrm{CI})$ & $(\mathrm{OR} / \mathrm{CI})$ \\
\hline LARC/ no LARC & 987 & $0.33(0.23-0.47)$ & $0.29(0.19-0.44)$ \\
\hline $\mathrm{LARC} / \mathrm{OC}^{\mathrm{a}}$ & 785 & $0.36(0.24-0.52)$ & $0.26(0.15-0.42)$ \\
\hline
\end{tabular}

${ }^{\mathrm{a}} \mathrm{OC}=$ Oral contraceptives, which includes combined oral contraceptives, and progestin-only pills.

$415{ }^{\mathrm{b}}$ The Chi-square test was used for calculating p-values and presented as Odds Ratios (OR) and 95\% confidence 416 intervals (CI).

$417{ }^{\mathrm{c}}$ A logistic regression model was used to adjust for age, previous pregnancies, childbirths and abortions. 